\title{
A NATURAL FORMULATION OF QUASI-INTERPOLATION BY MULTIVARIATE SPLINES
}

\author{
CHARLES K. CHUI AND HARVEY DIAMOND
}

\begin{abstract}
A quasi-interpolation formula based on discrete function values is given in the form of a multivariate spline series that yields the local approximation order characterized by the Fix-Strang conditions. This formula can be considered as a partial sum of the formal Neumann series expansion of the formal interpolation operator, and hence, justifies that "quasi-interpolation" is indeed an appropriate terminology for such an approximation formula.
\end{abstract}

1. Introduction. Although the exact order of best approximation by multivariate splines is still unknown in general, the local approximation order can be characterized by the so-called Fix-Strang conditions [6]. This characterization was formulated by Dahmen and Micchelli [5] in their study of controlled approximation, and recently de Boor and Jia [2] clarified controlled approximation as local approximation. In [3], Chui, Jetter, and Ward introduced the notion of the commutator of a locally supported multivariate spline function and showed that its degree is again equivalent to the Fix-Strang conditions. As an application, Chui and Lai [4] gave a quasi-interpolation scheme that gives the local approximation order.

In this paper, we present a quasi-interpolation formula, and using the abovementioned results, show that it also gives the local approximation order. Our formula can be considered as a partial sum of the formal Neumann series expansion of the formal interpolation operator. Hence, the terminology of quasi-interpolation first introduced by de Boor and Fix $[\mathbf{1}]$ is truly justified.

Let $\Phi$ be a locally supported piecewise polynomial function in $\mathrm{R}^{s}$ symmetric with respect to the origin, and $\hat{\Phi}$ its Fourier transform. Suppose that $\hat{\Phi}$ satisfies the Fix-Strang conditions

$$
\left\{\begin{array}{l}
\hat{\Phi}(0)=1, \\
D^{\alpha} \hat{\Phi}(2 \pi i)=0, \quad 0 \neq i \in \mathbb{Z}^{s},|\alpha| \leq \rho,
\end{array}\right.
$$

where $\rho$ is a nonnegative integer. Then $\rho$ is the local approximation order from the multivariate spline space spanned by all multi-integer translates of $\Phi$ (cf. [2 and 5]).

In obtaining a $\Phi$ of a given degree and smoothness, the largest $\rho$ is desirable. To accomplish this, in practice a linear combination $\phi$ of box splines of the required degree and smoothness is chosen which satisfies the linear equations (1) and yields

Received by the editors January 14, 1986.

1980 Mathematics Subject Classification (1985 Revision). Primary 41A15, 41A63.

Key words and phrases. Multivariate splines, local approximation order, quasi-interpolation, Neumann series.

This research was supported by the U.S. Army Research Office under Contract no. DAAG 29-84-K-0154. 
the maximal $\rho$ (cf. [5]). A finite number of translates of $\phi$ can be averaged to yield a symmetric $\Phi$ with the same local approximation order $\rho$ as $\phi$.

If $\mathbf{b}=\left\{b_{i}\right\}$ and $\mathbf{c}=\left\{c_{i}\right\}$, where $i \in \mathbf{Z}^{s}$, are two given sequences, we will use the standard convolution notation $\mathbf{b} * \mathbf{c}$ for the sequence $\left\{\sum_{j \in \mathbf{Z}} b_{j} c_{i-j}\right\}$ whenever the infinite series converges. We will also use the notation $\delta=\left\{\delta_{i 0}\right\}$, where $\delta_{i 0}=0$ if $i \neq 0$ and $\delta_{00}=1$.

Suppose that a continuous function $f$ on $\mathrm{R}^{s}$ is given. A multivariate spline series

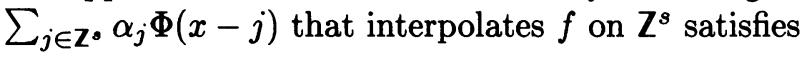

$$
\mathbf{M} * \boldsymbol{\alpha}=\mathbf{f}^{0},
$$

where $\alpha=\left\{\alpha_{i}\right\}, \mathbf{M}=\{\Phi(i)\}$, and $\mathbf{f}^{0}=\{f(i)\}$. Let

$$
\mathbf{M}=\boldsymbol{\delta}+\mathbf{m},
$$

where $\mathbf{m}=\left\{m_{i}\right\}$ with

$$
m_{i}= \begin{cases}\Phi(0)-1 & \text { for } i=0, \\ \Phi(i) & \text { for } i \neq 0 .\end{cases}
$$

Hence, if $\mathbf{M}$ would have an "inverse", then $\boldsymbol{\alpha}$ could be solved and a multivariate spline interpolant of $f$ could be determined. Unfortunately, the infinite linear system (2) does not have a unique solution for $\alpha$ in general. Nonetheless, let us consider the formal inverse of $\mathbf{M}$ and its formal Neumann series

$$
\mathbf{M}^{-1}=(\boldsymbol{\delta}+\mathbf{m})^{-1} \sim \boldsymbol{\delta}-\mathbf{m}+\cdots+(-1)^{k} \underbrace{\mathbf{m} * \cdots * \mathbf{m}}_{k}+\cdots .
$$

Its $k$ th partial sum defines a sequence of linear functionals on $C\left(\mathrm{R}^{s}\right)$ as follows:

$$
\left\{\left(\lambda_{k} f\right)(i)\right\}=(\boldsymbol{\delta}-\mathbf{m}+\cdots+(-1)^{k} \underbrace{\mathbf{m} * \cdots * \mathbf{m}}_{k}) * \mathbf{f}^{0} .
$$

Note that since $\Phi$ is locally supported, the convolution sums are actually finite sums, so that (4) is always defined for all $f$ in $C\left(\mathbf{R}^{s}\right)$. Our quasi-interpolation formulas will be given by

$$
\left(Q_{k} f\right)(x)=\sum_{j \in \mathbf{Z}^{*}}\left(\lambda_{k} f\right)(j) \Phi(x-j) .
$$

Again, since $\Phi$ is locally supported, the infinite series is actually a finite sum for each fixed $x$.

2. Preliminary lemmas. Let $\pi_{d}=\pi_{d}^{s}$ denote the space of all polynomials of total degree $\leq d$ in $s$ variables. The commutator of $\Phi$ with an $f$ in $C\left(\mathbf{R}^{s}\right)$ was defined in $[3]$ to be

$$
[\Phi \mid f]=\sum_{j \in \mathbf{Z}^{*}} \Phi(x-j) f(j)-\sum_{j \in \mathbf{Z}^{*}} \Phi(j) f(x-j) .
$$

It was defined to be of order $d+1$ or degree $d$ if $[\Phi \mid f]=0$ for all $f \in \pi_{d}$. Since $\hat{\Phi}(0)=1$ and $D^{\alpha} \Phi(2 \pi i)=0$ for all $i \in Z^{s} \backslash\{0\}$ and $|\alpha| \leq \rho$, it was shown in [3] that the commutator of $\Phi$ also has degree $\rho$, yielding the following result. 
LEMMA 1. If $g$ is in $\pi_{\rho}$, then the spline series

$$
\sum_{j \in \mathbf{Z}^{*}} g(j) \Phi(\cdot-j)
$$

is also in $\pi_{\rho}$.

We remark that this lemma also follows from a result in [2]. We also need the following lemma.

Let $\mathbf{Z}_{+}^{s}=\left\{i \in \mathbf{Z}^{s}: i \geq 0\right\}$.

LEMMA 2. For each positive integer $k$ and any $\alpha \in \mathbf{Z}_{+}^{s}$ with $|\alpha| \leq 2 k-1$,

$$
\underbrace{\mathbf{m} * \cdots * \mathbf{m}}_{k} *\left\{i^{\alpha}\right\}=0
$$

PROOF. Since $\Phi$ is locally supported and symmetric about the origin, and since $\sum m_{i}=0$, we can write

$$
\mathbf{m}=\sum_{p=1}^{N} \alpha_{p} \mathbf{l}(p)
$$

where $\alpha_{1} \ldots, \alpha_{N}$ are some constants and $\mathrm{l}(p)=\left\{l_{i}(p)\right\}$ with

$$
l_{i}(p)= \begin{cases}-2 & \text { for } i=0 \\ 1 & \text { for } i=i_{p} \text { or }-i_{p} \\ 0 & \text { otherwise }\end{cases}
$$

for some $i_{p} \in \mathbf{Z}^{s}$. In other words, $\mathbf{m}$ is a (finite) linear combination of certain second central differences. Since

$$
\mathrm{l}\left(p_{1}\right) * \cdots * \mathrm{l}\left(p_{k}\right) *\left\{i^{\alpha}\right\}=0
$$

for any $p_{1}, \ldots, p_{k}$ and $|\alpha| \leq 2 k-1$, the identity (6) follows.

The following result is of some interest by itself.

LEMMA 3. Let $f \in \pi_{2 k+1}$. Then $\left(Q_{k} f\right)(i)=f(i)$ for all $i \in \mathbf{Z}^{s}$.

Proof. By Lemma 2, we have

$$
\underbrace{\mathbf{m} * \cdots * \mathbf{m}}_{k+1} * \mathbf{f}^{0}=0 .
$$

Hence, it follows from (3), (4), and (5) that

$$
\begin{aligned}
& \left\{\left(Q_{k} f\right)(i)\right\}=\left(\lambda_{k} f\right) * \mathbf{M}=\left(\lambda_{k} f\right) *(\boldsymbol{\delta}+\mathbf{m}) \\
& =\left[(\boldsymbol{\delta}-\mathbf{m}+\cdots+(-1)^{k} \underbrace{\mathbf{m} * \cdots * \mathbf{m}}_{k})+(\mathbf{m}-\cdots+(-1)^{k} \underbrace{\mathbf{m} * \cdots * \mathbf{m}}_{k+1})\right] * \mathbf{f}^{0} \\
& \quad=\mathbf{f}^{0}+(-1)^{k} \underbrace{\mathbf{m} * \cdots * \mathbf{m}}_{k+1} * \mathbf{f}^{0}=\mathbf{f}^{0} .
\end{aligned}
$$

This completes the proof of the lemma. 
3. Main results. We are now ready to establish the main results of this paper.

THEOREM 1. Let $k$ be any nonnegative integer such that $\rho \leq 2 k+1$. Then $\left(Q_{k} f\right)=f$ for all $f \in \pi_{\rho}$.

REMARK 1. Of course, if only an optimal local approximant is required, the smallest integer $k$ should be chosen. Perhaps for large values of $k, Q_{k} f$ are "almost" interpolants of $f$ on $\mathbf{Z}^{s}$. This requires further investigation.

PROOF. Since $\left(\lambda_{k} f\right)(i)$ is a polynomial of $i \in \mathbb{Z}^{s}$ with total degree $\leq \rho$, we can conclude from Lemma 1 that $Q_{k} f$ is in $\pi_{\rho}$. From Lemma 3, we see that the two polynomials $Q_{k} f$ and $f$ agree on all of $\mathbb{Z}^{s}$, and hence, they must be identical. This completes the proof of the theorem.

Let $h>0$ and $h \mathbb{Z}^{s}=\left\{h j: j \in \mathbb{Z}^{s}\right\}$. As usual, we define the scaled quasiinterpolation operators $Q_{k}^{h}$ by

$$
\left(Q_{k}^{h} f\right)(x)=\sum_{j \in \mathbf{Z}^{s}}\left(\lambda_{k} f\right)(j h) \Phi\left(\frac{x}{h}-j\right) .
$$

Then by a standard proof in approximation theory, the following result can be obtained as a simple consequence of the above theorem.

THEOREM 2. Let $F$ be any compact set in $\mathrm{R}^{s}$ and $k \geq(\rho-1) / 2$ be any integer. Then there exists a positive constant $C_{F, k}$ such that for any integer $r \leq \rho$ and $f \in C^{r+1}(F)$

$$
\max _{x \in F}\left|\left(f-Q_{k}^{h} f\right)(x)\right| \leq C_{F, k}\left(\max _{x \in F} \sum_{|\alpha|=r+1}\left|D^{\alpha} f(x)\right|\right) h^{r+1} .
$$

REMARK 2. By a more careful investigation of the Fix-Strang conditions when $\Phi$ is determined, we could even obtain a multivariate order of approximation as studied in [4].

\section{REFERENCES}

1. C. de Boor and G. Fix, Spline approximatin by quasi-interpolants, J. Approx. Theory 8 (1973), 19-45.

2. C. de Boor and R. Q. Jia, Controlled approximation and a characterization of the local approximation order, Proc. Amer. Math. Soc. 95 (1985), 547-553.

3. C. K. Chui, K. Jetter, and J. D. Ward, Cardinal interpolation by multivariate splines, Math. Comp. (to appear).

4. C. K. Chui and M. J. Lai, A multivariate analog of Marsden's identity and a quasi-interpolation scheme, J. Constructive Approx. (to appear).

5. W. Dahmen and C. A. Micchelli, On the approximation order from certain multivariate spline spaces, J. Austral. Math. Soc. Ser. B 26 (1984), 233-246.

6. G. Strang and G. Fix, Fourier analysis of the finite element variational method, C.I.M.E. II Cilo 1971, Constructive Aspects of Functionl Analysis (G. Geymonat, Ed.), 1973, pp. 793-840.

Department of Mathematics, Texas a\&M University, College Station, TEXAS 77843

Department of Mathematics, West Virginia University, Morgantown, West VIRGINIA 25701 